\title{
Ambito de hogar y utilización de hábitat de dos grupos de venados Cola Blanca Odocoileus virginianus (Artiodactyla: Cervidae) reubicados en un ambiente tropical
}

\author{
Joel C. Sáenz y Christopher Vaughan
}

\begin{abstract}
Programa Regional en Manejo de Vida Silvestre para Mesoamérica y el Caribe. Universidad Nacional. Apartado Postal 1350-3000, Heredia, Costa Rica. Fax: 237-7039, Internet: jsaenz@una.ac.cr
\end{abstract}

Recibido 7-V-1998. Corregido 25-VI-1998. Aceptado 29-VII-1998

\begin{abstract}
Eight white-tailed deer were released in La Emilia farm, Guanacaste, Costa Rica, during November 1987 and March 1988. Four females were from an insular population (San Lucas Island, ISL) and had been raised in captivity up to 9 months of age when they were liberated. The remaining four were adults, three females and one male from Palo Verde National Park (PV). All deer were marked with radio telemetry collars and followed for a 13-month period. The four deer from ISL were also observed directly for 8 months. The home range and habitat use of each group was determined and compared. The average daily home range was 18.3 ha for the ISL group and 18.4 ha for the PV group; the differences between these values were not significant (Analysis of Variance, ANOVA, $P>.05$ ). The home ranges of the two groups were not significantly different between seasons; however the ISL group did show a significant difference between the two seasons (ANOVA, $\mathrm{P}<.05$ ). The ISL group used eight habitat types of the 14 available, while the PV group utilized 11 habitat types; differences were found in the habitat preferences between the two groups $\left(\mathrm{X}^{2} \mathrm{P}<.001\right)$. Habitat utilization was significantly different between the two groups in both the dry season and the wet season $\left(\mathrm{X}^{2}, \mathrm{P}<.001\right)$. The most utilized habitats (confidence intervals of Bonferroni) of the ISL group were grazing land, forest plantations (Pithecelobium saman), cultivated land (sorghum and fruits), and riparian vegetation. The PV group used habitats with certain forest cover, chaparral, jaraguales and guacimales (Guazuma ulmifolia).
\end{abstract}

Key words: White-tailed deer, Odocoileus virginianus, radiotelemetry, home range, habitat utilization, translocation, Costa Rica.

La reubicación ("translocación") y la reintroducción de especies de vida silvestre son herramientas de conservación con gran potencial para restaurar comunidades y ecosistemas naturales degradados por la actividad del hombre, tratando de aproximarse en lo posible al estado prístino de éstos (Stanley Price 1989). Sin embargo, muchos intentos para trasladar animales silvestres de un lugar a otro han fracasado y no es una técnica aplicable universalmente a todas las especies como muchos especialistas creen (Nielsen 1988).
Además, el traslado de animales silvestres de un hábitat a otro puede generar serios problemas, como una alta mortalidad de los animales reubicados, depresión genética por exogamia, riesgos de enfermedades, tanto para los animales que se liberan en el nuevo hábitat, como para la población residente del área de liberación, y pueden entrar en conflicto con el hombre y convertirse en plagas (Caldecott y Kavanagh 1988, Dodd y Siegel 1991, Templeton 1994, Cunningham 1996). Además de estos problemas de carácter biológico, las reubi- 
caciones son costosas, pueden generar conflictos políticos, y presentar problemas éticos (Fieldler et al 1993).

La reintroducción y/o reubicación de especies silvestres para el restablecimiento de sus poblaciones, ha sido uno de los mayores éxitos en el manejo de la vida silvestre en decadas pasadas y recientes (Taylor y Pelton 1979, Teer 1987). En Norteamérica, el venado cola blanca (Odocoileus virginianus) fue reintroducido y reubicado con gran éxito desde finales del siglo pasado y comienzos de éste; incrementándose las poblaciones de unos pocos a cientos de miles en la actualidad (Blackard 1971, Teer 1987). Durante un programa de reubicación muchos mamíferos silvestres no sobreviven o cambian sus patrones de comportamiento, porque son removidos de su sitio natal hacia otro ambiente totalmente desconocido, esto es más acentuado en aquellas especies que son filopátricas. Muchos animales reubicados tienden a regresar a su área natal recorriendo grandes distancias (>64 km), y algunos "vagan" en grandes áreas, recorriendo más de $350 \mathrm{~km}$ de distancia desde el sitio de liberación (Friits et al. 1988, Rogers 1988, Schmitt y Aho 1988).

El ámbito de hogar y uso de hábitat de venados cola blanca reintroducidos y/o reubicados, fueron estudiados principalmente en zonas templadas (Hamilton 1962, Marchinton y Jeter 1966, Hawkins y Montgomery 1969, Ozoga et al. 1992, Jones y Withman 1990). O'Bryan y McCuollough (1985), Temple y Evans (en prep.) estudiaron los movimientos de venados cola negra (Odocoileus hemionus) trasladados a nuevos hábitats. Según nuestro entendimiento, son escasos los estudios publicados sobre reubicación de venados cola blanca en Región Neotropical.

Los objetivos de este trabajo fueron: determinar el ámbito de hogar, y el uso del hábitat de dos grupos de venados (cervatillos criados en cautiverio y venados adultos silvestres) liberados en una finca agrícola-ganadera en Guanacaste, Costa Rica.

\section{MATERIALES Y MÉTODOS}

El estudio se realizó en tres áreas. La Isla San Lucas (ISL) fue el lugar donde se capturaron los cervatillos, la ISL se encuentra en el Golfo de Nicoya en la costa occidental de Costa Rica ( $9^{\circ} 56^{\prime}-9^{\circ} 57^{\prime} \mathrm{N}$ y $\left.84^{\circ} 53^{\prime}-84^{\circ} 55^{\prime} \mathrm{W}\right)$, cuya extensión es de 476 ha (Sáenz 1990). Una segunda área fue el Parque Nacional Palo Verde (PV), que se encuentra en la cuenca baja del Río Tempisque, Guanacaste $\left(10^{\circ} 19^{\prime}-10^{\circ} 24^{\prime} \mathrm{W}\right.$ y $\left.85^{\circ} 18^{\prime}-85^{\circ} 25^{\prime} \mathrm{W}\right)$, tiene una extensión de 16 804 ha (Sáenz 1990). En este lugar se capturaron los venados adultos silvestres. La tercera área fue la finca La Emilia, allí se criaron los cervatillos capturados en ISL, y el lugar donde se liberaron todos los animales de este estudio.

La finca La Emilia (FLE) ocupa una extensión de 5500 ha, y se encuentra en Cañas, provincia de Guanacaste, Costa Rica $\left(10^{\circ} 16^{\prime} 300^{\prime \prime}-10^{\circ} 22^{\prime} 45^{\prime \prime}\right.$ N y $85^{\circ} 04^{\prime} 05^{\prime \prime}$ 85013'00” W) (Sáenz 1990). La precipitación y temperatura promedio anual es de $1664 \mathrm{~mm}$ y $27.2^{\circ} \mathrm{C}$ respectivamente, siendo los meses de noviembre a abril los más secos (Sáenz 1990). La finca pertenece a las zonas de vida de bosque seco tropical y bosque seco tropical transición a húmedo (Tosi 1969).

El sitio de estudio tenía una extensión de 3 820 ha y ubicada en la parte oeste de la finca. El $60 \%$ de está área estuvo constituido por pastizales y cultivos agrícolas, principalmente caña de azúcar (Saccharum officinarum), sorgo (Sorghum bicolor), frutales como guanábana (Annona muricata) y mango (Mangifera indica). En la finca existía un hato de ganado de alrededor de 4000 cabezas. Los pastizales se encuentran asociados con hierbas, arbustos, grupos de árboles o una combinación de éstos. La mayoría de los pastizales se inundan periódicamente por efecto de las mareas, y también por las lluvias durante la estación húmeda. La cobertura boscosa en esta área está distribuida en: bosque semi caducifolio de bajura, bosque siempre verde de bajura y de montaña, manglares, parches aislados de vegetación, vegetación palustre y riparia (Sáenz 1990). 
Entre octubre de 1987 y febrero de 1988 se capturaron seis venados cola blanca adultos en el PV (grupo PV) con una red de caída (Sáenz 1990). El esfuerzo de captura fue de 26 días/hombre y 192 horas/trampa, con un costo de 150 dólares por venado capturado y liberado. Los venados fueron inmovilizados con $\mathrm{Xi}$ lacina (Rompun, Bayer, Leverkussen, Alemania) a una concentración del $2 \%(23.4 \mathrm{mg} / \mathrm{ml})$ e inyectada por vía intramuscular, con una dosis de aproximadamente $3 \mathrm{mg} / \mathrm{kg}$ por peso del animal.

Anestesiados los venados se les colocó un collar radiotransmisor (Telonics, Mesa, Arizona, 85204), posteriormente se registró el peso. Cada 15 min se le aplicaron compresas de agua fría por todo el cuerpo del animal para disminuir los efectos del anestésico, y en todo momento se observaron los signos vitales. Una hora después de ser inmovilizados los venados fueron transportados hacia FLE en cajas de madera laminada de $1.5 \mathrm{~m} \times 0.9 \mathrm{~m} \times 0.4 \mathrm{~m}$ (Sáenz 1990). Las liberaciones se hicieron en noviembre 1987 (2) y febrero de 1988 (4).

En la ISL se capturaron "a mano" 15 cervatillos de una a tres semanas de edad (grupo ISL), durante febrero y marzo de 1987. Cinco fueron criados en cautiverio en la FLE hasta los nueve meses de edad, y liberados juntos en noviembre de 1987. Estos venados también fueron pesados y marcados con collares radiotransmisores antes de ser liberados. Los dos grupos de venados fueron liberados en sitios diferentes a una distancia de $2.5 \mathrm{~km}$ uno del otro. El grupo PV en la parte sur de la FLE, y el grupo ISL frente al corral de crianza, ubicada en la parte noroeste de la finca. Dos hembras proveniente de PV murieron, una durante el traslado, y la otra tres días después de ser liberada. El cervato de ISL fue depredado por un coyote (Canis latrans) tres días posterior a la liberación. Por lo tanto, la muestra del estudio fue de ocho animales, cuatro de PV (tres hembras y un macho, adultos) y cuatro de ISL (hembras juveniles de 9 meses de edad).

Registro de datos: Se usó la técnica de radiotelemetría para la obtención de la mayoría de los datos de localización, para lo cual se usó un receptor TR-2 (banda de 150-152 Mhz) y una antena manual "H" RAK-2 (Telonics, Mesa, Arizona, 85204). Las localizaciones (coordenadas $\mathrm{x}, \mathrm{y}$ ) de los venados fueron obtenidos mediante triangulación (White y Garrot 1990).

Los acimuts (dos por cada localización) fueron registrados desde 130 estacas (estaciones) ubicadas sobre el terreno y separadas entre 100 y $350 \mathrm{~m}$, las cuales cubrían todo el sitio de estudio. El intervalo entre dos pares de acimuts consecutivos fue de $30 \mathrm{~min}$ para los dos grupos de venados, y el tiempo entre ambos acimuts fue inferior a los $5 \mathrm{~min}$. Los acimuts se determinaron con un error angular promedio de $2.5^{\circ}$.

Las cuatro venadas juveniles de ISL fueron monitoreados una vez por semana, durante un período de $24 \mathrm{hr}$ (06: 00-06: $00 \mathrm{hr}$ ó 18: 0018: $00 \mathrm{hr}$ del día siguiente, alternando estos horarios cada semana) desde noviembre de 1987 a noviembre de 1988. Para los cuatro venados del grupo PV el período fue de $12 \mathrm{hr}$ (06: 0018: $00 \mathrm{hr}$ o 18: 00-06: $00 \mathrm{hr}$ del día siguiente, alternando estos horarios cada semana), de noviembre de 1987 hasta noviembre de 1988 dos animales, y de febrero de 1988 a noviembre de 1988 dos hembras y un macho. Sin embargo, durante setiembre no se pudo recolectar datos para el grupo FV por problemas logísticos debido a los huracanes Gilbert y Joan que azotó Costa Rica (Anónimo 1988). Para ambos grupos, los registros se hicieron para cada animal.

Las coordenadas ( $\mathrm{x}, \mathrm{y})$ de las estaciones de registro de datos fueron obtenidas del mapa topográfico de la finca, mediante el uso de un digitalizador electrónico (GTCO, Corporation, Rockville, Maryland 20850) conectado a una micro computadora. Las localizaciones (coordenadas $\mathrm{x}, \mathrm{y}$ ) radiotelemétricas de los animales se obtuvieron con un programa para micro computadora escrito en Fortran V (Harestad 1981; Sáenz 1990). Este programa usó el valor de las coordenadas $(\mathrm{x}, \mathrm{y})$ de las estaciones, $\mathrm{y}$ los correspondientes acimuts registrados cada $30 \mathrm{~min}$.

Se elaboró un mapa de los hábitats del sitio de estudio a partir de fotografías aéreas, un 
mosaico controlado de fotografías aéreas, y un mapa topográfico de la finca. Los hábitats se clasificaron por asociaciones vegetales representativas basadas en el estado sucesional, florístico, fisiográfico, y por especies vegetales indicadoras (Sáenz 1990). El área de cada hábitat fue calculada usando el digitalizador electrónico.

Observación visual: Este método se usó con los venados de la ISL durante ocho meses (noviembre de 1987-Junio de 1988), cuando los animales fueron fáciles de observar. Se localizó cada animal con el equipo de radiotelemetría y se anotó su posición en el mapa de hábitat de la FLE. En la mayoría de los casos la distancia entre el observador y el animal no fue superior a los $50 \mathrm{~m}$. Para las localizaciones nocturnas de cada venado se usó una lámpara, siguiéndose el mismo método descrito anteriormente, en pocas ocasiones los venados alteraron su comportamiento natural cuando se uso este método. El valor de las coordenadas registradas por observación visual fue obtenido del mapa de la FLE, usando para ello el digitalizador electrónico.

Análisis de los datos: Las localizaciones de los venados fueron graficadas sobre el mapa de hábitat (escala: 1:9000) y analizadas por procedencia (ISL y PV). Los datos de cada grupo de venados fueron agrupados por día (24 hr). La independencia matemática entre coordenadas de localización sucesivas fue probada con la razón de Schoener: $\mathrm{t}^{2} / \mathrm{r}^{2}$ (Schoener 1981; Siwhart y Slade 1986). Para el cálculo de la razón de Schoener se usó una programa para micro computadora (Sáenz 1990).

$\mathrm{El}$ ámbito de hogar $(\mathrm{AH})$ diario fue estimado con coordenadas independientes para un período de $24 \mathrm{hr}$, y agrupados por grupos de venados. El AH mensual, por estaciones del año (eg. estación seca vs. lluviosa) y el AH total (todo el año) fueron calculados con todas las localizaciones independientes recolectadas en cada uno de estos periodos. Los $\mathrm{AH}$ fueron calculados por el método del polígono mínimo convexo (Mohr 1947), tomando el 100, 95 y $90 \%$ de las localizaciones y usando un programa para micro computadora (Harestad 1981, Sáenz 1990). Se efectuaron análisis de varianza para encontrar diferencias en las variaciones entre los $\mathrm{AH}$ diarios, mensuales, entre y dentro de las estaciones del año (seca y lluviosa), y entre los dos grupos de venados.

Para encontrar diferencias en el uso del hábitat de cada grupo de venados, se empleó Chicuadrado $\left(\mathrm{X}^{2}\right)$ (Neu et al. 1974). Para detectar que hábitat fue "seleccionado" o "evitado" (comparando. las proporciones de hábitats disponibles respecto de las proporciones utilizadas), se utilizaron los intervalos de confianza simultáneos de Bonferroni (Byers et al. 1984, Gibbons 1976, Neu et al. 1974). Una tabla de contingencia de $2 \mathrm{xN}$ ( $2=$ grupos de venados y $\mathrm{N}=$ tipos de hábitats) fue usada para determinar su hubo asociación o independencia entre los grupos de venados y los tipos de hábitat. Las localizaciones radiotelemétricas de cada grupo de venados en los diferentes hábitats fueron coordenadas independientes, agrupadas por estaciones, y para todo el estudio.

\section{RESULTADOS}

Ambito de Hogar (AH): El Cuadro 1 entrega el peso, sexo y AH promedio de cada venado a través de todo el período de estudio. La sobrevivencia fue del $66 \%$ para el grupo PV y $80 \%$ para el grupo ISL.

Los venados del grupo ISL aumentaron paulatinamente su AH después de ser liberados, alcanzando el valor máximo en marzo (penúltimo mes de la estación seca); después del cual el AH comenzó a disminuir hasta mostrar un patrón más o menos estable entre julio y noviembre (estación lluviosa) (Fig. 1). En cambio, el grupo PV mostró poca variación en el tamaño del AH diario con respecto al grupo ISL (ANDEVA p> .05). El grupo PV registró AH mayores en los primeros meses posteriores a la liberación, para luego decrecer durante los meses de la estación lluviosa (Fig. 1).

El tamaño promedio del AH fue menor en la estación seca que en la estación lluviosa para venados del grupo ISL, esta diferencia fue significativa a nivel de los tres porcentajes considerados para la estimación del AH (ANDEVA $\mathrm{p}<.05)$. Sin embargo, los venados del 


\section{CUADRO 1}

Ambito de hogar diario (AHD), y distancia diaria recorrida (DRD) de ocho venados liberados en la finca La Emilia, Guanacaste, Costa Rica

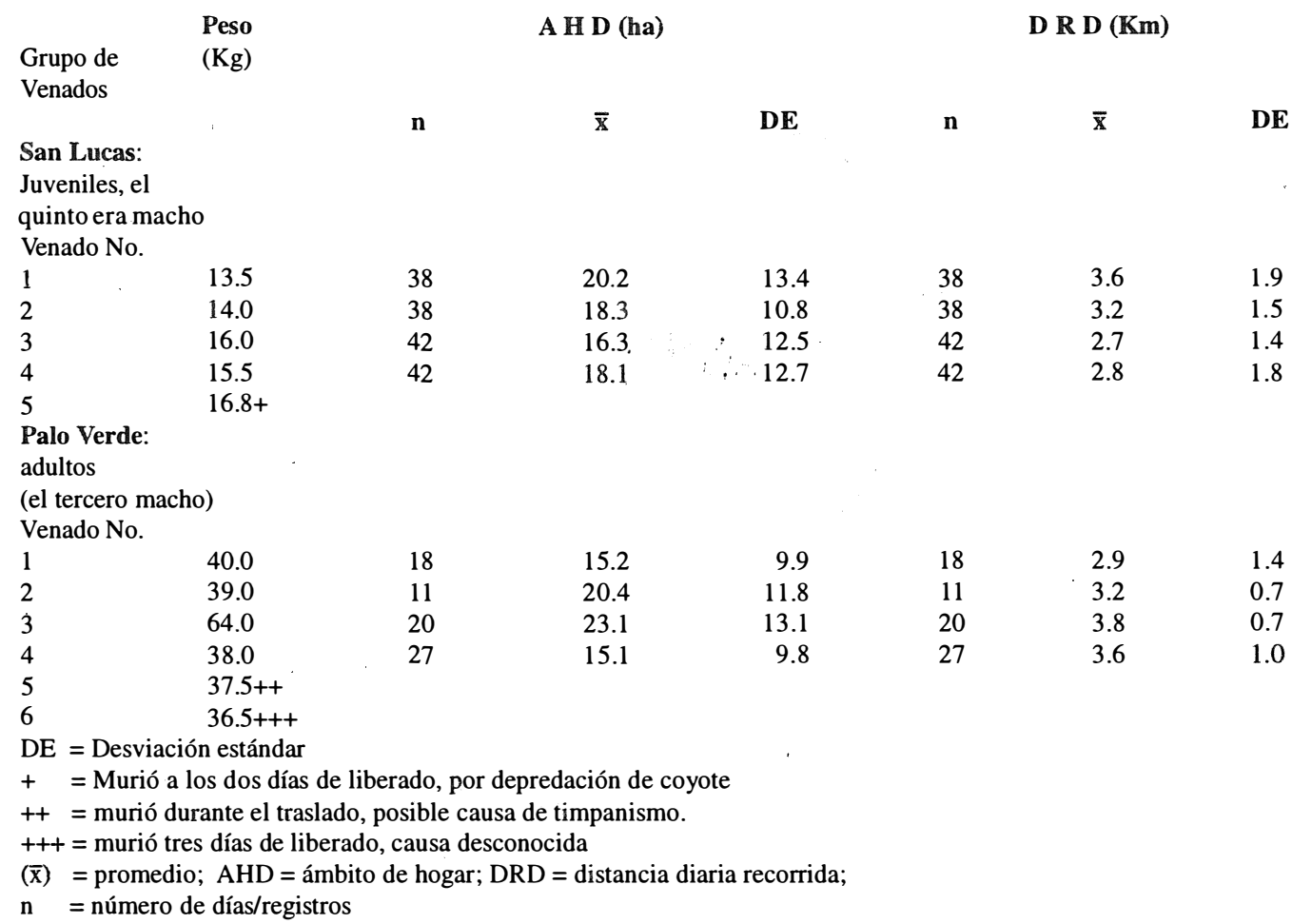

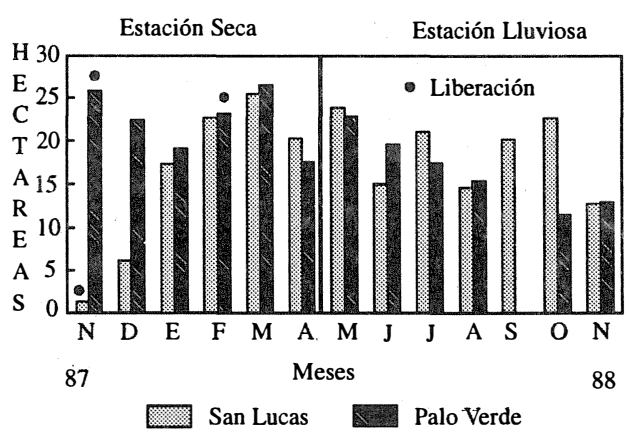

Fig. 1. Ambito de hogar promedio diario (24 horas) agrupados por mes de dos grupos de venados cola blanca (San Lucas y Palo Verde) liberados en la Finca La Emilia, Guanacaste, Costa Rica.1987-1988.

grupo $\mathrm{PV}$ tuvieron un $\mathrm{AH}$ promedio más grande durante la estación seca. La diferencia en el $\mathrm{AH}$ promedio entre las dos estaciones del año fue significativa entre los dos grupos $(90 \%$ de las localizaciones), siendo el AH del grupo ISL mayor al del grupo PV (ANDEVA $p<.05$ ). El AH total promedio (anual) para el grupo ISL fue de 509 ha (334.4-754.3) mientras que para el grupo PV fue de solo 317.2 ha (159.4510.6). Sin embargo, no se encontraron diferencias significativas (Kruskall-Wallis $\mathrm{p}>.05$ ).

Utilización y selección de hábitat: Se determinaron 14 tipos de hábitats (Cuadro 2 y 3 ), de los cuales siete pueden considerarse como "artificiales", estos son: pastizales, cañaverales, frutales, plantaciones forestales de cenízaro (Pithecelobium saman), jaraguales (Hyparrhenia rufa), cultivos agrícolas y áreas de influencia humana; los restantes se consideraron naturales, como manglares, guacimales ( $\mathrm{Gua}$ zuma ulmifolia); vegetación riparia y palustre; bosques caducifolio, semicaducifolio y perennifolio. Los datos de radiotelemetría y observación visual indicaron que los venados usaron 


\section{CUADRO 2}

Intervalos de confianza simultáneos de Bonferroni (ICB) para la utilización de los diferentes tipos de hábitats, por venados de la Isla San Lucas (ISL) y Palo Verde (PV) reubicados en la finca La Emilia, Guanacaste,

Costa Rica, durante la estación seca y húmeda. 1987-1988

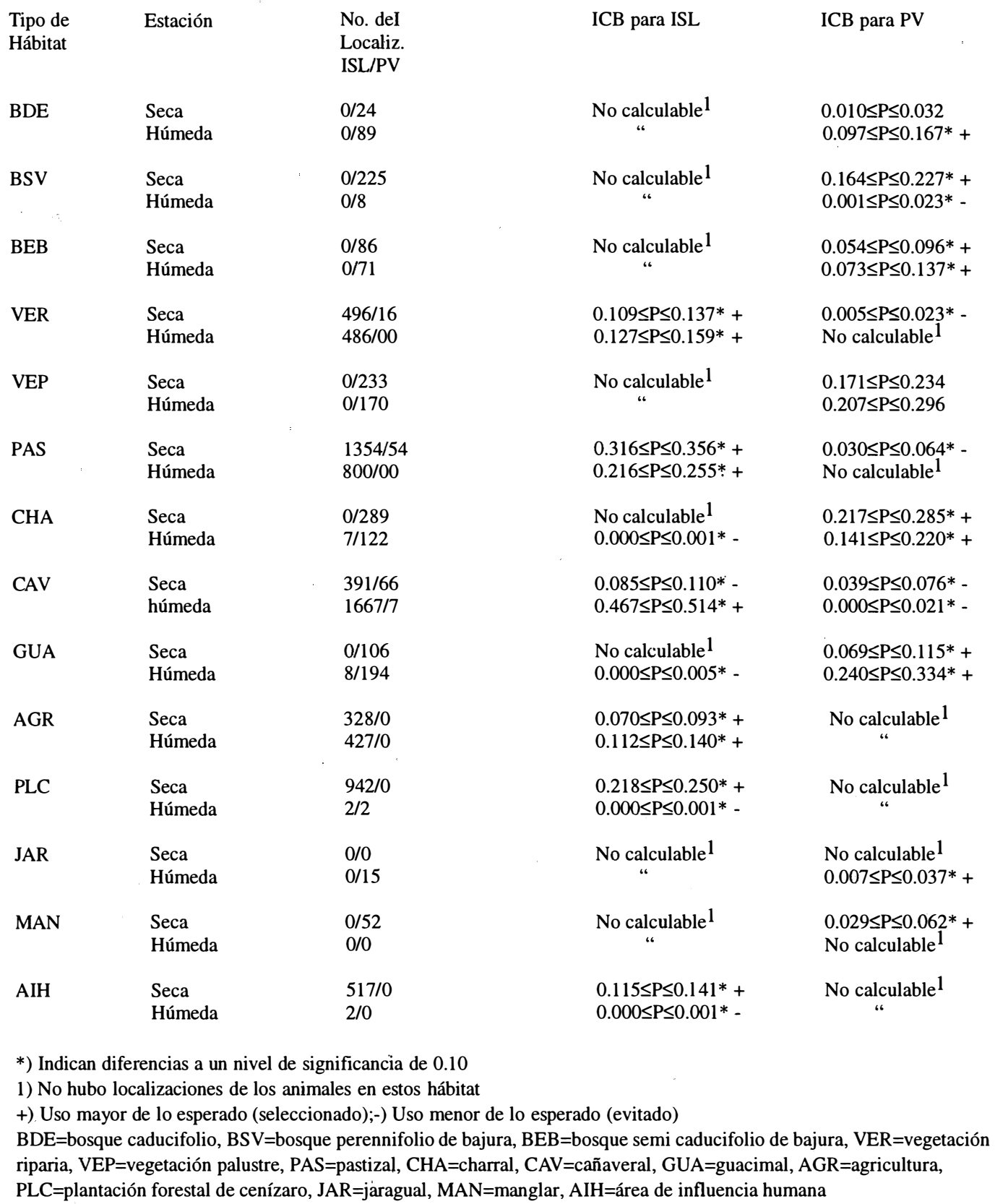




\section{CUADRO 3}

Intervalos de confianza simultáneos de Bonferroni para la utilización de diferentes tipos de hábitats $(\mathrm{Pi})$ por venados de Isla San Lucas (ISL) y Palo Verde (PV) reubicados en la finca La Emilia, Guanacaste, Costa Rica. Los datos son para todo el período de estudio (1987-1988)

\begin{tabular}{|c|c|c|c|c|c|}
\hline $\begin{array}{l}\text { Tipo de } \\
\text { Hábitat }\end{array}$ & $\begin{array}{l}\text { Grupos de } \\
\text { Venados }\end{array}$ & $\begin{array}{l}\text { Número de } \\
\text { Localizaciones }\end{array}$ & $\begin{array}{l}\text { Proporción } \\
\text { Calculada (Pio) } \\
\text { de uso }\end{array}$ & $\begin{array}{l}\text { Proporción } \\
\text { Esperada de Uso } \\
\text { (Pi) }\end{array}$ & $\begin{array}{l}\text { Intervalos } \\
\text { Para (Pi) }\end{array}$ \\
\hline $\mathrm{BDE}$ & $\begin{array}{l}\text { ISL } \\
\text { PV }\end{array}$ & $\begin{array}{l}0 \\
113\end{array}$ & $\begin{array}{l}0 \\
0.062\end{array}$ & $\begin{array}{l}0.028 \\
0.028\end{array}$ & $\begin{array}{l}\text { No calculable } \\
0.047 \leq \mathrm{P} \leq 0.077^{*}\end{array}$ \\
\hline BSV & $\begin{array}{l}\text { ISL } \\
\text { PV }\end{array}$ & $\begin{array}{l}0 \\
243\end{array}$ & $\begin{array}{l}0 \\
0.133\end{array}$ & $\begin{array}{l}0.036 \\
0.036\end{array}$ & $\begin{array}{l}\text { No calculable } 1 \\
0.112 \leq \mathrm{P} \leq 0.154^{*}+\end{array}$ \\
\hline BEB & $\begin{array}{l}\text { ISL } \\
\text { PV }\end{array}$ & $\begin{array}{l}0 \\
157\end{array}$ & $\begin{array}{l}0 \\
0.086\end{array}$ & $\begin{array}{l}0.051 \\
0.051\end{array}$ & $\begin{array}{l}\text { No calculable } 1 \\
0.068 \leq \mathrm{P} \leq 0.104^{*}+\end{array}$ \\
\hline VER & $\begin{array}{l}\text { ISL } \\
\text { PV }\end{array}$ & $\begin{array}{l}988 \\
16\end{array}$ & $\begin{array}{l}0.125 \\
0.009\end{array}$ & $\begin{array}{l}0.034 \\
0.034\end{array}$ & $\begin{array}{l}0.115 \leq \mathrm{P} \leq 0.135^{*}+ \\
0.003 \leq \mathrm{P} \leq 0.015^{*}-\end{array}$ \\
\hline VEP & $\begin{array}{l}\text { ISL } \\
\text { PV }\end{array}$ & $\begin{array}{l}0 \\
396\end{array}$ & $\begin{array}{l}0 \\
0.217\end{array}$ & $\begin{array}{l}0.218 \\
0.218\end{array}$ & $\begin{array}{l}\text { No calculable } 1 \\
0.191 \leq \mathrm{P} \leq 0.243\end{array}$ \\
\hline PAS & $\begin{array}{l}\text { ISL } \\
\text { PV }\end{array}$ & $\begin{array}{l}2614 \\
45\end{array}$ & $\begin{array}{l}0.331 \\
0.025\end{array}$ & $\begin{array}{l}0.194 \\
0.194\end{array}$ & $\begin{array}{l}0.317 \leq \mathrm{P} \leq 0.345^{*}+ \\
0.015 \leq \mathrm{P} \leq 0.034^{*}-\end{array}$ \\
\hline $\mathrm{CHA}$ & $\begin{array}{l}\text { ISL } \\
\text { PV }\end{array}$ & $\begin{array}{l}7 \\
411\end{array}$ & $\begin{array}{l}0.000 \\
0.225\end{array}$ & $\begin{array}{l}0.029 \\
0.029\end{array}$ & $\begin{array}{l}0.000 \leq \mathrm{P} \leq 0.000^{*}- \\
0.199 \leq \mathrm{P} \leq 0.251^{*}+\end{array}$ \\
\hline $\mathrm{CAV}$ & $\begin{array}{l}\text { ISL } \\
\text { PV }\end{array}$ & $\begin{array}{l}2058 \\
73\end{array}$ & $\begin{array}{l}0.261 \\
0.040\end{array}$ & $\begin{array}{l}0.356 \\
0.356\end{array}$ & $\begin{array}{l}0.247 \leq \mathrm{P} \leq 0.274 * \\
0.028 \leq \mathrm{P} \leq 0.052 *\end{array}$ \\
\hline GUA & $\begin{array}{l}\text { ISL } \\
\text { PV }\end{array}$ & $\begin{array}{l}12 \\
300\end{array}$ & $\begin{array}{l}0.002 \\
0.164\end{array}$ & $\begin{array}{l}0.007 \\
0.007\end{array}$ & $\begin{array}{l}0.000 \leq \mathrm{P} \leq 0.003^{*} \\
0.141 \leq \mathrm{P} \leq 0.187^{*}+\end{array}$ \\
\hline AGR & $\begin{array}{l}\text { ISL } \\
\text { PV }\end{array}$ & $\begin{array}{l}755 \\
0\end{array}$ & $\begin{array}{l}0.096 \\
0\end{array}$ & $\begin{array}{l}0.003 \\
0.003\end{array}$ & $\begin{array}{l}0.087 \leq \mathrm{P} \leq 0.105^{*}+ \\
\text { No calculable }\end{array}$ \\
\hline PLC & $\begin{array}{l}\text { ISL } \\
\text { PV }\end{array}$ & $\begin{array}{l}944 \\
0\end{array}$ & $\begin{array}{l}0.120 \\
0\end{array}$ & $\begin{array}{l}0.004 \\
0.004\end{array}$ & $\begin{array}{l}0.110 \leq \mathrm{P} \leq 0.129^{*}+ \\
\text { No calculable }\end{array}$ \\
\hline JAR & $\begin{array}{l}\text { ISL } \\
\text { PV }\end{array}$ & $\begin{array}{l}0 \\
22\end{array}$ & $\begin{array}{l}0 \\
0.012\end{array}$ & $\begin{array}{l}0.005 \\
0.005\end{array}$ & $\begin{array}{l}\text { No calculable } \\
0.005 \leq \mathrm{P} \leq 0.019\end{array}$ \\
\hline MAN & $\begin{array}{l}\text { ISL } \\
\text { PV }\end{array}$ & $\begin{array}{l}0 \\
52\end{array}$ & $\begin{array}{l}0 \\
0.028\end{array}$ & $\begin{array}{l}0.027 \\
0.027\end{array}$ & $\begin{array}{l}\text { No calculable } \\
0.018 \leq \mathrm{P} \leq 0.039\end{array}$ \\
\hline $\mathrm{AIH}$ & $\begin{array}{l}\text { ISL } \\
\text { PV }\end{array}$ & $\begin{array}{l}517 \\
0\end{array}$ & $\begin{array}{l}0.065 \\
0\end{array}$ & $\begin{array}{l}0.008 \\
0.008\end{array}$ & $\begin{array}{l}0.058 \leq \mathrm{P} \leq 0.073^{*}+ \\
\text { No calculable }\end{array}$ \\
\hline
\end{tabular}

*) Indican diferencias a un nivel de significancia del 0.10 .

1) No hubo localizaciones de los animales en estos hábitat.

+) Uso mayor que lo esperado (seleccionado), -) Uso menor de lo esperado (evitado)

$\mathrm{BDE}=$ bosque caducifolio, $\mathrm{BSV}=$ bosque perennifolio, $\mathrm{BEB}=$ bosque semi caducifolio de bajura, VER=vegetación riparia, $\mathrm{VEP}=$ vegetación palustre, $\mathrm{PAS}=$ pastizal, $\mathrm{CHA}=$ charral, $\mathrm{CAV}=$ cañaveral, $\mathrm{GUA}=$ guacimal, $\mathrm{AGR}=$ agricultura, $\mathrm{PLC}=\mathrm{planta}$ ción forestal de cenízaro, JAR=jaragual, MAN=manglar, AIH=área de influencia humana. 
los hábitats de manera diferencial, siendo estas diferencias muy significativas para cada grupo de venado durante todo el estudio $\left(\mathrm{X}^{2} \mathrm{p}<.001\right)$. El grupo de venados de ISL utilizó más el cañaveral y el grupo PV uso pequeños parches de guácimo, con un 54 y $67 \%$ de las localizaciones respectivamente (Figs. 2 y 3 ).

Tipos de Habitad
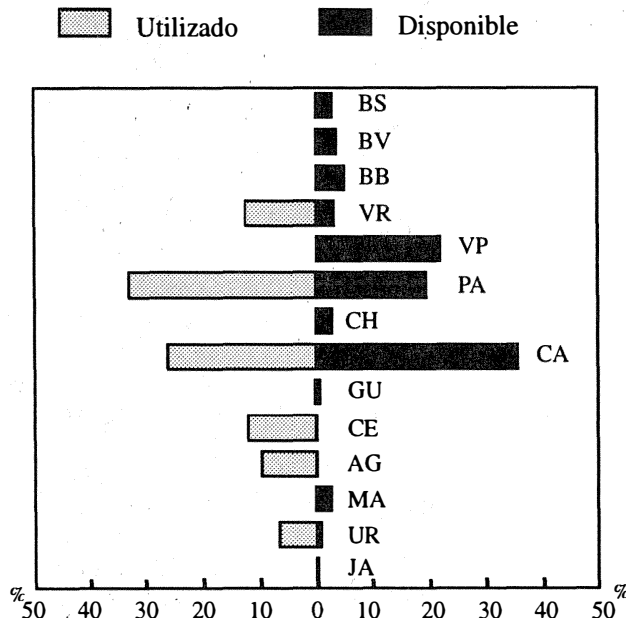

Fig. 2.Porcentaje de hábitat disponible y porcentaje de localizaciones en cada tipo de hábitat de venados cola blanca de la Isla San Lucas, liberados en la Finca La Emilia, Guanacaste, Costa Rica, 1987-1988. AG=agricultura, BS=bosque caducifoluio, $\mathrm{BB}=$ bosque semicaducifolio de bajura, $B V=$ perennifolio de bajura, $C A=$ cañaveral, $C E-$ =plantación forestal de cenízaro, $\mathrm{CH}=$ charral, $\mathrm{GU}=$ guacimal, JA=jaragual, $M A=$ manglar, $V R=$ vegetación ribereña, $\mathrm{VP}=$ vegetación palustre, $\mathrm{PA}=$ pastizal, $\mathrm{UR}=a$ rea de influencia humana.

Se encontraron diferencias significativas $\left(\mathrm{X}^{2} \mathrm{p}<.001\right)$ cuando se comparó el uso del hábitat entre los dos grupos de venados (ISL y PV) durante la estación seca. También hubo diferencias significativas entre los dos grupos en la utilización del hábitat durante la estación lluviosa $\left(\mathrm{X}^{2} \mathrm{p}<.001\right)$. Las Figs. 2 y 3 muestran el grado de utilización que hicieron los venados de cada uno de los diferentes tipos de hábitats considerados (utilización vs hábitat disponible).
Tipos de Habitat

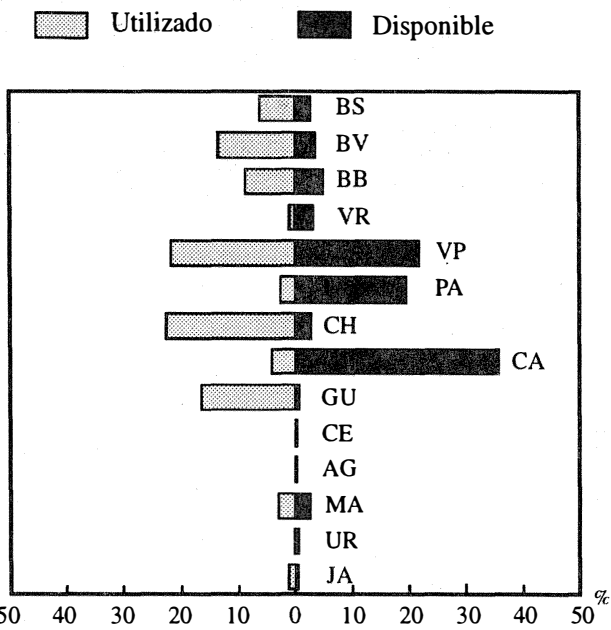

Fig. 3.Porcentaje de hábitat disponible y porcentaje de localizaciones en cada tipo de hábitat de venados cola blanca de Palo Verde, liberados en la Finca La Emilia,Guanacaste, Costa Rica, 1987-1988. AG=agricultura, BS=bosque caducifoluio, $\mathrm{BB}=$ bosque semicaducifolio de bajura, $\mathrm{BV}=$ perennifolio de bajura, $\mathrm{CA}=$ cañaveral, $\mathrm{CE}=$ plantación forestal de cenízaro, $\mathrm{CH}=$ charral, $\mathrm{GU}=$ guacimal, JA=jaragual, $M A=$ manglar, $V R=$ vegetación ribereña, $V P=$ evegetación palustre, $\mathrm{PA}=$ pastizal, $\mathrm{UR}=$ área de influencia humana.

La vegetación riparia, los pastizales, y cultivos agrícolas fueron hábitats seleccionados por los venados del grupo ISL (utilizaron los hábitats en mayor proporción de lo que estuvieron disponibles) en ambas estaciones del año. El cañaveral fue evitado durante la época seca, pero seleccionado en el época lluviosa. Hábitats como plantaciones de cenízaro y áreas de influencia humana fueron seleccionados en la estación seca y evitado en la estación lluviosa por este grupo (Cuadro 2). El grupo PV seleccionó los charrales, guacimales y el bosque semi caducifolio de bajura, tanto en la estación lluviosa como en la estación seca, mientrás que el jaragual no fue utilizado en la estación seca, pero fue seleccionado en la estación lluviosa. Este mismo grupo seleccionó el bosque siempre verde en la estación seca, pero evitado en la estación húmeda (Cuadro 2). 
Tomando en cuenta todas las localizaciones de cada grupo de venados, la utilización del hábitat mostró diferencias entre los dos grupos $\left(\mathrm{X}^{2} \mathrm{p}<.001\right)$. Los hábitats seleccionados en la estación seca y húmeda, también fueron importantes al término del estudio. Sin embargo, el cañaveral fue la excepción, este hábitat fue evitado por los venados del grupo ISL a pesar de contener 2058 (26.4\%) de las localizaciones totales (cuadro 3), y representar el $35 \%$ de la superficie total de los hábitats.

\section{DISCUSIÓN}

Los venados cola blanca reubicados en nuevas áreas tienden a "vagar" sin un patrón definido, recorriendo largas distancias y registrando AH más grandes que en su lugar de origen, principalmente durante los primeros meses post-liberación, debido a que pasan por un período de exploración y aprendizaje en nuevo hábitat antes de fijar un AH definitivo (Hamilton 1962, Marchinton y Jeter 1966, Nichols y Whitehead 1978, O'Bryan y McCullough 1985). La mayor sobrevivencia de venados los juveniles de este estudio también fue reportado para zonas templadas (Hamilton 1962, Jones y Witham 1990, Marchinton y Jeter 1967), dichos autores señalan que los cervatillos y juveniles son el mejor material genético para usar en los programas de reubicación o reintroducción, debido a que se adaptan con mayor facilidad al nuevo ambiente.

Los AH promedio diarios de los individuos del grupo ISL fueron similares entre ellos durante los primeros ocho meses, y se debió principalmente al comportamiento de "manada" que mostraron durante este período, este comportamiento es común en venados que son criados en cautiverio y luego liberados (Siegler 1968). Sin embargo, estos valores fueron semejantes a los señalados por Marchinton y Jeter (1966) para venados semi silvestres en Norte América.

No se sabe como los cambios estacionales del ambiente afectan los movimientos diarios de venados reubicados, pero si está bien docu- mentado la inufluencia de estos cambios en venados residentes de varias zonas de Norte América. Estas variaciones no implican cambios en los límites del AH, pero sí en los patrones de movimientos, y parece estar relacionado con la disponibilidad de alimentos (Hood 1979, Kammeryer y Marchinton 1976, Marchinton y Hirth 1984).

El AH del grupo ISL en la estación lluviosa fue mayor que en la época seca. Atribuímos esta diferencia a disturbios climáticos, como los huracanes Gilbert y Joan (IMN 1988) que inundaron más del $50 \%$ del área de estudio en setiembre y octubre, provocando una disminución en la disponibilidad del forraje debido a su inmersión. Estas variaciones del AH coinciden con lo señalado para venados residentes en Norte América, donde los AH son más grandes durante los períodos de escasez de alimentos y condiciones climáticas severas (Larson et al. 1978, Rongstad y Tester 1969).

No obstante, estos disturbios probablemente no afectaron mucho a los venados del grupo PV, porque las áreas que ocuparon no se inundaron totalmente quedando pequeños "islotes" donde los venados pasaron los días de inundación, reduciendo notablemente sus movimientos y utilizando los recursos básicos para sobrevivir, similar situación observaron Sparrowe y Springer (1970) con venados residentes en noroeste de los Estados Unidos.

La fecha de liberación pudo ser otro factor que contribuyó al menor tamaño del AH del grupo ISL durante la estación seca. Esta fecha coincidió con el inicio de esta estación, y durante los primeros meses los animales no se alejaron mucho del sitio de liberación (corral); incluso, durante los dos primeros meses regresaron frecuentemente a dormir al corral de crianza; por lo tanto, tuvieron muy poca actividad nocturna, esta situación poco usual en condiciones de comportamiento natural del venado cola blanca contribuyó a que el $\mathrm{AH}$ en esta época fuera pequeño.

El AH total anual fue mayor en el grupo ISL (509 ha) que en el grupo PV (317.2 ha); esta diferencia también fue observada en zonas templadas, donde venados juveniles silvestres 
y/o criados en cautiverio siempre exhibieron mayores movimientos y ocuparon más área durante la post-liberación, que venados adultos residentes o reubicados (Jones y Whitman 1990, Ozoga et al. 1992).

Calvopiña (1994) determinó un AH anual promedio de 323.2 ha para venadas silvestres reubicadas de ISL a tierra firme (Cóbano, Puntarenas, Costa Rica), este valor del $\mathrm{AH}$ anual fue semejante al de los venados del grupo PV. Esta similitud de valores en los $\mathrm{AH}$, pudo ser causada entre otras cosas por factores bioclimáticos (temperatura, lluvia, etc.) que comparten ambas áreas. Cóbano y FLE se encuentran en la misma zona geográfica y pertenecen a la zona de vida bosque seco tropical, además poseen tipos de hábitats similares (Calvopiña 1994, Sáenz 1990); por lo tanto, es de esperar que los venados silvestres reubicados dentro de esta misma zona geográfica, muestren similitudes en los patrones de movimientos.

Sin embargo, el sitio donde fueron liberadas las hembras del grupo de la ISL estuvo rodeada de pastizales con ganado, y la actividad humana fue mayor; además, eran animales jóvenes. Estos factores pudieron haber retardado el establecimiento del $\mathrm{AH}$, porque tuvieron que invertir más tiempo en explorar y adaptarse al nuevo hábitat, similar situación ocurrió con venados juveniles reubicados en Norte América (Hamilton 1962, Marchinton y Jeter 1966).

El venado cola blanca está adaptado a una variedad de hábitats, tales como bosques, charrales, pantanos, pastizales, áreas de cultivo y áreas con actividad humana (Halls 1980, Hamilton y Whitaker 1979, Hardin et al. 1984). La selección en la estación seca por los pastizales, plantaciones de cenízaro y cultivos agrícolas del grupo ISL puede explicarse por:

a) "disponibilidad de alimento": la mayoría de los pastizales en La FLE fueron regados periódicamente por acción de las mareas, por lo tanto siempre hubo de forraje disponible; además, existió forraje resistente a la sequía como Sida spp. y Corchorus spp., especies apetecidas por estos venados (Espach y Sáenz 1994), además exitía plantaciones de cenízaro (cerca del punto de liberación) eran jóvenes y el sotobosque ofreció mucho forraje que los venados consumen, además de cobertura para termoregular; y b) "sitio de liberación": el sitio donde se liberó este grupo estuvo rodeado de pastizales en un radio de $1 \mathrm{~km}$, además la liberación coincidió con la estación seca, período en el cual los animales se estaban adaptando al nuevo hábitat; y al tener los venados un comportamiento tipo "homing" (Rogers 1988) los animales no se dispersaron mucho y utilizaron intensamente estos hábitats.

En la estación lluviosa los venados de la ISL seleccionaron las plantaciones de caña de azúcar, debido a la escasez de alimentos en los potreros por efecto de las inundaciones causados por los huracanes, en los cañaverales áreas los niveles de agua bajaron más rápidamente; por lo tanto, hubo más alimento disponible en .este tipo de hábitat que en los potrerose hábitat.

Los venados del grupo PV seleccionaron hábitats con remanentes boscosos durante la estación seca, similar comportamiento fue observado por Irby y Calvopiña (1994) en Cóbano, donde venados silvestres reubicados usaron hábitats con cierta cobertura boscosa, a diferencia de venados criados en cautiverio. En la estación seca, la disponibilidad de agua y alimento disminuyó en la FLE, lo cual obligó a los animales a desplazarse hacia hábitats más productivos como las áreas boscosas. En estos hábitats, hay fuentes alternativas de alimentos como frutos, forraje fresco $y$, existen pequeños manantiales de agua; por lo tanto, se esperaría que los venados usen intensamente dichos hábitats.

Este hecho explicaría en parte, porqué en los meses de la estación seca el AH diario fue mayor que en la época lluviosa. Similar situación ocurrió en Norte América, que ante condiciones ambientales severas, los venados ampliaron su ámbito de hogar y buscaron forraje y protección en otras áreas (Larson et al. 1978, Mooty et al. 1987, Hirht 1977). Durante la estación lluviosa los venados del grupo PV seleccionaron asociaciones de guácimo, charral, bosque seco y bosque semi caducifolio. Los guacimales, charrales, y el bosque caducifolio son hábitats utilizados por los venados en la 
ISL y el PV, porque ofrecen cobertura y forraje de alta calidad durante esta época del año (Rodríguez et al. 1985, Rodríguez 1989).

Es común el uso de pastizales por el venado cola blanca (Brokx y Andressen 1970, Gallina 1984, Hansen y Reid 1975). Aunque no presentamos datos sobre la dieta de los venados, la utilización de pastizales por los venados en la FLE no apunta hacia una competencia potencial con el ganado, pero puede ocurrir en épocas de gran escasez de recursos alimentarios. La dieta de los venados del grupo ISL estuvo constituida por plantas que el ganado no consume; además, muchas de estas plantas son tóxicas para el ganado (Sáenz, datos sin publicar).

\section{RESUMEN}

Se liberaron ocho venados cola blanca (Odocoileus virginianus) en finca La Emilia, Guanacaste, Costa Rica entre noviembre de 1987 y 1988; cuatro hembras juveniles de una población insular (Isla San Lucas, ISL) fueron criadas en cautiverio y liberadas a los nueve meses de edad. Los restantes fueron adultos: tres hembras y un macho procedentes del Parque Nacional Palo Verde (PV). Todos los venados fueron marcados con collares radiotransmisores y monitoreados por 13 meses. La observación visual se uso por ocho meses para los venados de la ISL. Se determinó el ámbito de hogar (AH) y utilización del hábitat de los venados liberados. El AH promedio diario fue de 18.2 ha para el grupo de ISL y 18.5 para los venados de PV; no se encontraron diferencias entre los $\mathrm{AH}$ entre grupos (Análisis de Varianza ANDEVA, $\mathrm{P}>$.05). Los AH estacionales no presentaron diferencias significativas; sin embargo, el grupo ISL sí mostró diferencias entre las dos estaciones (ANDEVA, $\mathrm{P}<.05$ ). El grupo de ISL utilizó ocho tipos de hábitats, de los 14 disponibles y, el grupo de PV 11. Los dos grupos de venados utilizaron los hábitats de manera diferencial $\left(\mathrm{X}^{2}, \mathrm{P}<.001\right)$. La utilización de hábitat fue diferente entre los grupos durante la época seca y durante la estación húmeda $\left(\mathrm{X}^{2}, \mathrm{P}<.001\right)$. Los hábitat seleccionados (Intervalos de Confianza de Bonferroni) por los venados de ISL fueron plantaciones forestales (Pithecelobium saman), cultivos agrícolas (sorgo y frutales), área urbana, vegetación riparia y pastizales; para el grupo de PV fueron guacimales (Guazuma ulmifolia), charrales, áreas con cobertura boscosa y jaraguales.

\section{AGRADECIMIENTOS}

Agradecemos al Fondo Mundial para la Vida Silvestre (WWF), La Organización para
Estudios Tropicales (OET), La Universidad Nacional y a la Ganadera La Emilia por financiar este proyecto. A Holly Espach por su ayuda en la colección de los datos de campo. A Jaime Rau y Miguel Rodríguez por los comentarios a este manuscrito.

\section{REFERENCIAS}

Anónimo. 1988.. Huracán Gilbert y Joan. Reporte climatológico de Noviembre. Instituto Metereológico Nacional, San José, Costa Rica. 25 p.

Anderson, D.T. 1979. The effect of dog harassment on translocated white-tailed deer (Odocoileus virginianus) in the Cumberland Plateau in Tennessee Tech. Rep. No 79-8. Tennessee Wildlife Resources Agency. Nashville, Tennessee. 78 p.

Blackard, J. 1971. Restoration of white-tailed deer in the Southwestern United States. Unpubl. M.S. thesis. Lousiana State University. Baton Rouge, Lousiana. 167 p.

Brokx, P.A. \& F.M. Andressen. 1970. Análisis estomacales del venado caramerudo de los llanos venezolanos. Bol. Soc. Venez. Cien. Nat. 27: 330-353.

Byers, R.C., R.K. Steinhorst \& P.R. Krausman. 1984. Clarification of a technique for analysis of utilizationavailability data. J.Wildl. Manage. 48(3): 1050-1053.

Caldecott, J.O \& M. Kavanagh. 1988. Strategic guidelines for nonhuman primate translocation, p. 64-75. In $\mathrm{L}$. Nielsen y R. Brown (eds.). Traslocations of Wild Animals. The Wisconsin Humane Society, Inc. and The Caesar Kleberg Wildlife Research Institute, Wisconsin and Texas.

Calvopiña, J. 1994. Evaluación de la reintroducción del venado cola blanca en Cóbano, Puntarenas, Costa Rica, p. 369-381. In C. Vaughan y M. Rodríguez (eds.). Ecología y Manejo del Venado Cola Blanca en México y Costa Rica. EUNA, Universidad Nacional, Heredia, Costa Rica.

Cunningham, A. 1996. Disease risk of wildlife translocations. Conser. Biol. 10(2): 349-353.

Dodd, C.K. \& R.A. Siegel. 1991. Relocations, repatriation, and translocation of amphibians and reptiles: are they conservation strategies that work?. Herpetology 47(3): 336-350.

Espach, H. \& J.C. Sáenz. 1994. Comportamiento de cervatos criados en cautiverio y reintroducidos en la finca 
La Emilia, Costa Rica, p. 163-179. In C. Vaughan y M. Rodríguez (eds.). Ecología y Manejo del Venado Cola Blanca en México y Costa Rica. EUNA, Universidad Nacional, Heredia, Costa Rica.

Fieldler, P.L., R.A. Laven, R.D. Gershenz \& L. Saul. 1993. The contemporary paradigm in ecology and its implications for endangered species conservation. End. Spec. Upd. 10 (3 y 4): 7-12.

Fritts, S.H., W.J. Paul \& L.D. Mech. 1988. Movements of traslocated wolves in Minnesota, p. 123-141. In L. Nielsen y R. Brown (eds.). Traslocations of Wild Animals. The Wisconsin Humane Society, Inc. and The Caesar Kleberg Wildlife Research Institute, Wisconsin and Texas.

Gallina, S. 1984. Ecological aspects of the coexplotation of deer Odocoileus virginianus and cattle. Act. Zool. Fennica 172: 251-254.

Gibbons, J.D. 1976. Nonparametric Methods for Quantitative Analysis. Holt, Rinehart and Winston, Nueva York. 445 p.

Halls, L.K. 1980. White-tailed deer, p. 43-63. In J. Schimidt y D.L. Gilbert (eds.). Big Game of North America: Ecology and Management. Stackpole, Harrisburg, PA.

Hamilton, R. 1962. Factors affecting dispersal of deer released in Indiana. J. Wildl. Manage. 26(1): 79-85.

Hamilton, W.J. \& J.O. Whitaker. 1979. Mammals of the Eastern United States. Cornell University, Ithaca, Nueva York. 456 p.

Hansen, R.M. \& D. Reid. 1975. Diet overlap of deer, elk and cattle in southern Colorado. J. Range. Manage. 28(1): 43-47.

Hardin, J.W., W.D. Klimstra \& N.J. Silvy. 1984. Florida Key, p. 381-390. In Halls, L.K. (ed.). White-tailed Deer: Ecology and Management. Stackpole, Harrisburg. Pensilvania.

Harestad, A.S. 1981. Computer analysis of home range data. Fish andWildl. Branch. Bull. No. B-11. Ministry of Environment. Columbia, Canada.

Hawkins, R.E. \& G.C. Montgomery. 1969. Movements of translocated deer as determined by telemetry data. J. Wildl. Manage: 3(1): 196-203.

Hirth, D.H. 1977. Social behavior of white-tailed deer in relation to hábitat. Wildl. Monogr. No 53. The Wildlife Society, Washington, D.C. 55 p.
Hood, R.E. 1979. Seasonal variations in home range, diel movements and activity patterns of white-tailed deer on the Rob and Bessie Welder Wildlife Refuge. M.Sc. Thesis, Texas A \& M University, College Station, Texas. $173 \mathrm{p}$.

Irby, L. \& J. Calvopiña. 1994. Uso de hábitat por el venado cola blanca reintroducido en la Península de Nicoya, Costa Rica, p. 333-346. In C. Vaughan y M. Rodríguez (eds.). Ecología y manejo del venado cola blanca en México y Costa Rica. EUNA, Universidad Nacional, Heredia, Costa Rica.

Jones, J.M. \& J.H. Witham. 1990. Post-translocation survival and movements of metropolitan white-tailed. Wildl. Soc. Bull. 18: 434-441.

Kammeryer, K.E. \& R.L. Marchinton. 1976. Notes on dispersal of male white-tailed deer. J. Mamm. 57(4): 776-778.

Larson, T.J., O.J. Rongstad \& F.W. Terbilcox. 1978. Movement and habitat use of white-tailed deer in South Central Wisconsin. J. Wildl. Manage. 42(1): 113-117.

Marchinton, R.L. \& L.K. Jeter. 1966. Telemetric study of deer movement ecology in the Southeast. Proc. AnnConf. S. E. Assoc. Fish \& Wildl. Comm. 20: 189-205.

Marchinton, R.L \& D.H. Hirth. 1984. Behavior, p. 129-168. In L. K. Halls (ed.). White-tailed Deer, Ecology and Management. Stackpole, Harrisburg, Pensilvania.

Mohr, C. 1947. Table of equivalent populations of North American small mammals. Am. Midl. Nat. 37: 223-249.

Mooty, J.J., P.D., Karns \& T.K. Fuller. 1987. Habitat use and seasonal range size of white-tailed deer in north central Minnesota. J. Wild. Manage. 51(3) : 644-648.

Neu, C.W., C.R. Byers \& J.M. Peek. 1974. A technique for analysis of utilization-availability data. J. Wildl. Manage. 38(3): 541-545.

Nichols, R.G. \& C.J. Whitehead. 1978. Effects of dog harrassment on relocated white-tailed deer. Proc. Ann. Conf. S. E. Assoc. Fish \&Wildl. Agencies 32: 195-201

Nielsen, L. 1988. Definitons, considerations, and guidelines for traslocation of wild animales, p. 12-51. In $\mathrm{L}$. Nielsen \& R. Brown (eds.). Traslocations of Wild Animals. The Wisconsin Humane Society, Inc. and The Caesar Kleberg Wildlife Research Institute. Wisconsin and Texas.

O’Bryan, M.K \& D.R. McCullough. 1985. Survival of black-tailed deer following relocation in California. J. Wildl. Manage. 49(1): 115-119. 
Ozoga, J.J., R.V. Doepker \& R.D. Earle. 1992. Hunter-harvest of captive-raised male white-tailed deer, Odocoileus virginianus, released in upper Michigan. Can. Field Natur. 106(3): 357-360.

Rogers, L.L. 1988. Homing tendencies of large mammals: A review, p. 76-92. In L. Nielsen and R. Brown (eds.). Traslocations of Wild Animals. The Wisconsin Humane Society, Inc. and The Caesar Kleberg Wildlife Research Institute, Wisconsin and Texas.

Rodríguez, M.A., C. Vaughan, V. Villalobos, y M. McCoy. 1985. Notas sobre el movimiento del venado colablanca (Odocoileus virginianus rafinesque) en un bosque seco tropical de Costa Rica. EUNED, Universidad Estatal a Distancia, San José, Costa Rica. $103 \mathrm{p}$.

Rodríguez, M.A. 1989. Tamaño y composición de los grupos sociales del hato de venados cola blanca (Odocoileus virginianus) de la Isla San Lucas, Costa Rica. Tesis de Licenciatura, Universidad Nacional, Heredia, Costa Rica. 62 p.

Rongstad, O.J. \& J.R. Tester. 1969. Movements and habitat use of white-tailed deer in Minnesota. J. Wild. Manage. 33(2): 366-379.

Sáenz, J.C. 1990. Ecología de dos grupos de venados (Odocoileus virginianus) liberados en un nuevo hábitat. Tesis de Licenciatura, Universidad Nacional, Heredia, Costa Rica. 135 p.

Schmitt, S. \& R. W. Aho. 1988. Reintroduction of moose from Ontario to Michigan, p. 258-274. In L. Nielsen y R. Brown (eds.). Traslocations of Wild Animals. The Wisconsin Humane Society, Inc. and The Caesar Kleberg Wildlife Research Institute, Wisconsin and Texas.

Schoener, T.W. 1981. An empirically based estimate of home range. Theorical Population Biology 20: 281-325.
Siegler, H.R. 1968. Life history of deer in New Hampshire, p. 53. In H.R. Siegler. (ed.). The white-tailed deer of New Hampshire. New Hampshire Fish and Game Departament. $185 \mathrm{p}$.

Sparrow, R.D \& P.F. Springer. 1970. Seasonal activity patterns of white-tailed deer in eastern South Dakota. J. Wild. Mange. 34(2): 420-430.

Stanley Price, M.R. 1989. Animal Re-introductions: The Arabian Oryx in Oman. Cambridge University, Cambridge. $291 \mathrm{p}$.

Swihart, R.K. \& N.A. Slade. 1986. The importance of statistic power when testing for independence in animal movements. Ecology 67: 255-258.

Taylor, C.I. \& M.R. Pelton. 1979. Evaluation of a raccoon translocation attempt in east Tennessee. Proc. Ann. Conf. S. E. Assoc. Fish and Wildl. Agencies. 33: 187-194.

Teer, J. 1987. Aspectos socioeconómicos del venado cola blanca en Texas, p. 19-22. In V. Solís, M. Rodríguez \& C. Vaughan (eds.). Actas del Primer Taller Sobre el Venado Cola blanca (Odocoileus virginianus) del Pacífico Seco. Universidad Nacional. Heredia, Costa Rica.

Templeton, A.R. 1994. Coadaptation local adaptation, and outbreeding depression, p. 152-153. In G.K. Meffe y C.R. Carroll. Principles of Conservation Biology. Sinauer, Sunderland, Massachusetts.

Tosi, J. 1969. Mapa ecológico de Costa Rica. Escala 1: 751)000. Centro Científico Tropical. San Jose, Costa Rica.

White, G.C \& R.A. Garrot. 1900. Analysis of Wildlife Radio-tracking Data. Academic, San Diego, California. $383 \mathrm{p}$. 